Service social

\title{
Réactions québécoises à l'État-providence en émergence : perspectives historiques
}

\section{Peter Southam}

Volume 38, numéro 2-3, 1989

Les politiques sociales

URI : https://id.erudit.org/iderudit/706436ar

DOI : https://doi.org/10.7202/706436ar

Aller au sommaire du numéro

Éditeur(s)

École de service social de l'Université Laval

ISSN

1708-1734 (numérique)

Découvrir la revue

Citer cet article

Southam, P. (1989). Réactions québécoises à l'État-providence en émergence : perspectives historiques. Service social, 38(2-3), 161-178.

https://doi.org/10.7202/706436ar
Résumé de l'article

Dans une perspective historique, l'auteur montre comment la notion d'État-providence a pris racine au Québec entre le début des années 1930 et le milieu des années 1960. En examinant les deux principales thèses sur lesquelles s'appuient les interprétations les plus courantes, soit la thèse du retard et la thèse de la modernisation sans heurt, l'auteur analyse les paramètres du discours tenu pendant trois décennies pour montrer comment l'expérience québécoise est à la fois ressemblante mais aussi divergente des autres sociétés occidentales. 


\section{ARTICLES}

Southam, Peter, professeur d'histoire au Département de sciences humaines de I'Université de Sherbrooke.

\section{Réactions québécoises à l'État-providence en émergence : perspectives historiques}

\section{Peter Southam}

Faut-il parler d'une crise de l'État-providence en cette fin du vingtième siècle? Depuis quelques années, un nombre croissant d'intervenants ont donné foi à cette notion. En même temps il apparaît que les diagnostics qui sous-tendent les constats de crise divergent et se contredisent au point où l'observateur se demande si la véritable crise ne se situerait pas au niveau des définitions et des perceptions plus que dans les structures mêmes du régime. Une chose est certaine : ceux qui remettent en question les acquis de l'État-providence profitent du fait que les systèmes d'idées ayant favorisé l'émergence, il y a vingt-cinq ans, d'un large consensus sur les objectifs des politiques de bien-être, ont perdu de leur force et paraissent moins pertinents aujourd'hui dans un nouveau contexte de valeurs, normes et rapports sociaux associés aux sociétés postindustrielles. 
En examinant comment, dans le passé, différents types de sociétés ont procédé à la remise en question et à la redéfinition des politiques sociales, I'historien peut contribuer à clarifier les enjeux contemporains en ce domaine. Dans cette perspective, le problème des conditions qui ont entouré et guidé l'élaboration du concept d'État-providence prend aujourd'hui un intérêt tout particulier. Le présent article examinera comment la notion d'État-providence a pris racine au Québec entre le début des années 1930 et le milieu des années 1960. Nous présenterons quelques-unes des conclusions majeures d'une étude plus élaborée portant sur l'ensemble des courants de pensée québécois, touchant ce sujet ${ }^{1}$. Les contraintes d'espace nous obligent à adopter une approche synthétique qui s'articulera en trois parties. La première partie examinera I'historiographie québécoise des politiques sociales. Nous présenterons ensuite les "paramètres » ou éléments constants du débat. Enfin la dernière partie traitera des oppositions et des ruptures entre courants de pensée qui ont marqué et qui en fin de compte expliquent le passage d'une définition résiduelle vers une définition institutionnelle du bien-être social ${ }^{2}$.

\section{L'historiographie des politiques sociales québécoises}

Les perspectives à partir desquelles l'historien examine l'évolution des politiques sociales se sont transformées à mesure que le développement des connaissances a modifié notre appréciation de la nature des transformations structurelles et culturelles qui ont affecté la société québécoise. Depuis les années 1950, deux grandes thèses sur le changement social au Québec ont marqué l'historiographie des politiques sociales : la thèse du retard et la thèse de la modernisation sans heurt.

\section{La thèse du retard}

En ce qui a trait aux politiques sociales, I'historiographie québécoise demeure fortement influencée par des interprétations qui datent de l'époque de la Révolution tranquille et qui mettent l'accent sur les thèmes de retard ou de rattrapage. Ces interprétations nous sont venues des nouvelles élites qui, au début des années 1960, ont joué un rôle central dans l'importante refonte des cadres institutionnels alors en cours. De tels auteurs avaient naturellement tendance à exagérer l'anachronisme des institutions existantes de façon à 
légitimer de nouvelles institutions en voie d'élaboration. Pour comprendre dans quelle mesure la théorie du retard a influencé l'interprétation de l'histoire des politiques sociales au Québec, il est nécessaire d'en rappeler brièvement les grandes lignes.

La thèse du retard prend pour acquis qu'il s'était produit une rupture entre les dimensions structurelles et culturelles de l'organisation sociale québécoise. Alors que les structures auraient évolué à un rythme comparable à ce qui se produisait dans les sociétés nordaméricaines ambiantes (le Québec aurait traversé les phases décisives de l'industrialisation au tournant du siècle), la culture et en particulier les mentalités et les représentations idéologiques ne se seraient pas transformées au même rythme ${ }^{3}$. Ce décalage entre industrialisation et culture serait attribuable au fait que les secteurs économiques d'avant-garde se trouvaient sous contrôle étranger. Mais plus fondamentalement, ce décalage aurait pour cause le contrôle qu'exerçait le clergé au sein même de la société franco-québécoise et qui avait pour effet de bloquer la modernisation et, par extension, la reprise en mains de l'économie par les Franco-Québécois. Une variante plus récente de cette thèse met l'accent sur le rôle de la petite bourgeoisie traditionnelle dont l'alliance avec le clergé aurait été cimentée par la hantise des changements socio-économiques et culturels; ces deux groupes auraient perçu la modernisation comme mettant en danger leurs intérêts propres. Il faut retenir qu'au-delà de l'idée d'un retard de la société franco-québécoise par rapport aux sociétés ambiantes, cette thèse met l'accent sur un retard de certains rouages de l'organisation sociale par rapport à d'autres. De là, l'image d'une société souffrant d'une rupture pathologique entre des réalités objectives découlant de son statut de société industrialisée d'une part, et le monde des représentations qui, d'autre part, semble bloqué à I'heure pré-industrielle. Cette perspective contribua évidemment à donner à la Révolution tranquille l'image rassurante d'un rattrapage, la mutation se présentant avant tout comme une révolution culturelle qui aurait permis une normalisation longtemps retardée.

\section{Le retard des politiques sociales}

C'est par référence à ce schéma général d'interprétation du changement social au Québec que la plupart des historiens des politiques sociales québécoises ont défini leur sujet. II est significatif à cet égard que les jugements développés en 1963 par le Rapport Boucher fassent encore autorité un quart de siècle plus tard ${ }^{4}$. Ce rapport, officiellement intitulé Rapport du comité d'étude sur l'assis- 
tance publique, a appliqué rétrospectivement à son analyse de la genèse du système québécois de sécurité sociale une vision institutionnelle et développementale du bien-être qui venait tout juste d'acquérir un statut central dans le discours des spécialistes. Compte tenu de cette problématique de départ, il n'est pas surprenant que, constatant la juxtaposition des programmes en place et la survie d'agences privées à côté des instances gouvernementales, le rapport finisse par présenter le régime québécois de bien-être comme une anomalie historique. Dans l'optique du rapport, il aurait été normal, compte tenu du caractère industrialisé de la province de Québec, que le gouvernement ait depuis longtemps assumé pleine responsabilité dans le secteur du bien-être afin de mettre en œuvre une politique sociale complète et intégrée. Comment expliquer que des institutions québécoises ne se soient pas développées de cette façon ? Selon les auteurs du Rapport Boucher, la faute revenait à la doctrine sociale de l'Église : "cet arrière-plan philosophique explique pourquoi le transfert graduel de la responsabilité privée à celle du secteur public ne s'est pas fait systématiquement, à partir d'une volonté bien définie et exprimée dans un plan d'ensemble $»^{5}$. En d'autres termes, un décalage entre la situation socio-économique réelle et la réponse institutionnelle à cette situation serait surtout attribuable à l'influence du clergé.

Le récent ouvrage d'Yves Vaillancourt, L'évolution des politiques sociales au Québec: 1940-1960, représente une contribution de grande importance qui demeurera une des références essentielles pour I'histoire des programmes sociaux au Québec et au Canada ${ }^{6}$. Le sujet qui nous intéresse ici s'y trouve évidemment beaucoup plus approfondi que dans le Rapport Boucher. L'auteur met notamment en évidence l'influence des rapports de classes : I'alliance entre la petite bourgeoisie traditionnelle et le clergé, les supports communs de la petite bourgeoisie franco-québécoise et du grand capital au régime de I'Union nationale. II faut néanmoins constater que les grandes lignes de l'interprétation coïncident avec celles du Rapport Boucher. L'inertie du gouvernement québécois en matière de politique sociale est présentée comme une aberration, particulièrement quand l'auteur l'oppose à ce qu'il définit comme une attitude infailliblement positive du gouvernement fédéral dans ce domaine. Vaillancourt avance que le gouvernement Duplessis aurait dû " utiliser constructivement et activement l'État provincial, à la manière d'un levier, pour planifier le développement social et soutenir énergiquement les familles populaires aux prises avec des problèmes aigus... ${ }^{7}$ Le fait que la Saskatchewan et la Colombie-Britannique aient développé des programmes innovateurs en matière de politique sociale est présenté comme une preuve que le Québec aurait pu faire autant ${ }^{8}$. 


\section{La thèse de la modernisation sans heurt}

Depuis une dizaine d'années, la plupart des études spécialisées touchant le changement social au Québec tendent à remettre en question la thèse du retard. Caldwell et Czarnocki ont notamment fait valoir que cette thèse aurait exagéré le décalage entre les facettes structurelles et culturelles de l'organisation sociale franco-québécoise en limitant l'analyse des structures au seul taux d'industralisation sans $d^{\prime}$ ailleurs tenir compte du type $d^{\prime}$ industrialisation concerné ${ }^{9}$. Ces auteurs ont mis en lumière le fait que des transformations structurelles extrêmement importantes s'étaient produites au cours des années 1950 au plan des moyens de communication, de la démographie, des structures socio-professionnelles et de l'urbanisation. Les changements culturels associés à la Révolution tranquille apparaissent dès lors comme une réponse aux changements structurels beaucoup plus qu'un rattrapage. Les œuvres de synthèse historique tendent actuellement à mettre l'accent sur la conformité de l'expérience du Québec par rapport aux sociétés ambiantes. Comme I'a souligné Fernand Ouellet, l'étude récente de Linteau-Durocher-Robert-Richard présente "l'entrée du Québec dans une ère de modernité [comme s'étant produit] à peu près en même temps qu'ailleurs et dans des circonstances similaires ${ }^{10}$. Pour Ouellet, la thèse d'une modernisation sans heurt se présente comme presque aussi réductrice que la thèse du retard car selon lui, elle ne tient pas suffisamment compte de deux spécificités québécoises : d'une part, les profondes différences qui séparent l'expérience de la société montréalaise de celles des sociétés régionales et, d'autre part, les différences entre l'expérience des Franco-Québécois et celle des autres Québécois.

Ce n'est que récemment que la thèse de la modernisation sans heurt a commencé à influencer I'histoire des politiques sociales. Les auteurs qui écrivent dans cette perspective prennent note de la réticence du Québec face aux initiatives fédérales, mais ils font surtout ressortir que la pression des besoins et l'opinion publique avaient amené l'État québécois à intervenir de façon ponctuelle de sorte que, du point de vue fonctionnel, le régime québécois de bienêtre n'avait pas grand-chose à envier aux régimes en vigueur à la même époque dans les provinces et états voisins ${ }^{11}$. Enfin, suivant cette interprétation, l'État-providence n'arrive à maturité que dans les années 1960 et 1970 au Québec comme dans les sociétés ambiantes. C'est l'époque où une thématique de "guerre à la pauvreté " et de "société juste" mobilise toute l'Amérique du Nord ${ }^{12}$. 


\section{Au-delà des deux thèses en présence}

Il est difficile d'être entièrement satisfait de l'un ou de l'autre de ces deux schémas d'interprétation de l'histoire des politiques sociales au Québec. En ce qui concerne la thèse du retard, nous partageons I'avis des critiques qui maintiennent que le Québec n'a pas souffert de décalage significatif même si son système de bien-être était incontestablement différent de ceux des sociétés ambiantes. L'histoire administrative d'autres gouvernements, notamment celle du gouvernement canadien, démontre que ce ne fut qu'exceptionnellement que les pouvoirs publics ont abandonné une attitude négative vis-à-vis l'élargissement du rôle social de l'État.

Les auteurs qui se sont penchés sur les politiques sociales d'Ottawa sont unanimes à insister sur le conservatisme du gouvernement fédéral ${ }^{13}$. Avant 1960 ce dernier ne s'était jamais engagé politiquement en faveur d'une définition institutionnelle du bien-être du type prôné par le Rapport Boucher comme " allant de soi ». Même si pendant la deuxième guerre, des comités d'enquête (Marsh, Heagerty) avaient appuyé une telle définition, les gouvernements King, Saint-Laurent et Diefenbaker s'en sont tenus à une conception résiduelle du bien-être fondée sur la prémisse que les programmes sociaux ne devraient s'appliquer que lorsque la famille et l'économie de marché seraient incapables de subvenir aux besoins. Les premiers signes d'une volonté politique résolument institutionnelle - annonciatrice des grandes initiatives des années 1960 et 1970 - apparaîtront lors du congrès tenu en 1960 à Kingston par le Parti libéral fédéral alors à l'opposition.

Il y eut bien sûr les expériences de la Saskatchewan et de la Colombie-Britannique, mais ici les conditions socio-économiques sous-jacentes et les problèmes sociaux qui en découlaient avaient si peu en commun avec la situation qui prévalait dans les provinces plus industrialisées que, de toute évidence, ces modèles politiques n'étaient pas transférables.

En ce qui concerne maintenant la thèse de la modernisation sans heurt, nous considérons que cette approche ne tient pas suffisamment compte des conflits et des contradictions particulières qu'impliquait le processus de modernisation pour une société comme le Québec. Une société dominée par des puissances économiques ou politiques étrangères risque de voir la modernisation renforcer les rapports de dépendance. Dans les sections suivantes, nous montrerons comment cette situation particulière explique qu'aux yeux des élites québécoises, les enjeux de la redéfinition des politiques sociales n'étaient pas tout à fait les mêmes qu'ailleurs. 
Dans leur façon de situer l'expérience québécoise par rapport au mouvement général de redéfinition des institutions qui a affecté l'Occident tout entier entre 1930 et 1960 et qui a mené à la maturation de l'État-providence, les deux interprétations examinées ici semblent partager une conception essentiellement déterministe du changement social selon laquelle chaque société devrait suivre les mêmes étapes sur une trajectoire société traditionnelle/société moderne. À l'encontre de cette conception, I'histoire comparée des politiques sociales tend à suggérer que les expériences varient considérablement selon les sociétés et que ce manque de convergence est attribuable à des spécificités structurelles et culturelles locales.

\section{Les paramètres du discours}

Au Québec, comme ailleurs, deux points de référence ont guidé I'élaboration des politiques sociales : d'une part, la pensée économique normative ou l'évolution de la science économique; d'autre part, la référence au concept de communauté. L'ambivalence des politiques sociales, leur caractère toujours controversé, s'expliquent en grande partie par l'incompatibilité de ces deux niveaux de définition qui ont chacun tendance à pousser les politiques sociales vers des finalités différentes.

\section{À l'échelle occidentale}

Dans l'ensemble des pays occidentaux comme au Québec, le premier de ces points de référence a dominé historiquement : les politiques sociales ne se sont développées que dans les interstices laissés par une "saine pensée économique ". ${ }^{14}$ Pour sa part, le concept de communauté fournit un cadre de référence plus constructif qui permet de contrecarrer l'influence limitative de la théorie économique. En rapport avec ce deuxième point de référence, il est possible de voir l'histoire des politiques sociales comme un effort continu pour renouveler ou restaurer la réalité sociale de communauté sans cesse mise en péril par les transformations structurelles de l'économie. C'est d'ailleurs seulement dans la mesure où un ensemble d'individus ou de groupes partagent un sentiment d'appartenance à une même collectivité au sein de laquelle la vie et l'intérêt des membres sont associés à la vie et à l'intérêt de l'ensemble que ces individus développeront un sentiment d'obligation face à autrui et le sens de l'altruisme nécessaire à l'essor de politiques sociales progressistes. ${ }^{15}$ Sans ces sentiments, les individus et les groupes n'accepteraient jamais les transferts unilatéraux en biens et services 
qu'impliquent généralement les politiques sociales. La définition des politiques sociales doit évidemment tenir compte de ces deux cadres de référence. Mais dans le processus d'élaboration, une tension s'établit inévitablement entre ces deux points, et chaque société doit construire son ordre de priorités en tenant compte de sa situation concrète.

\section{À l'échelle québécoise}

Pour traiter maintenant plus particulièrement du cas francoquébécois, nous avons constaté que le discours tenu entre 1930 et 1960 sur le sujet des politiques sociales a évolué au rythme de la science économique d'une part, et d'autre part, à celui de la redéfinition du concept de communauté. Le discours franco-québécois suit en ceci une tendance générale propre à l'ensemble des sociétés occidentales. Mais en même temps, les élites francoquébécoises ont dû tenir compte de la spécificité de la situation historique à laquelle ils avaient à faire face. Les impératifs de la question nationale en particulier ont imposé un itinéraire implacable au processus de redéfinition des politiques sociales.

En ce domaine, une différence primordiale sépare l'expérience franco-québécoise de celle des sociétés les plus souvent traitées dans I'historiographie des politiques sociales. Alors que dans les autres sociétés, la définition des politiques sociales a surtout répondu à des impératifs de fonctionnalité interne, c'est en regardant à la fois vers l'intérieur et vers l'extérieur que les élites franco-québécoises ont abordé cette question. Dans les sociétés occidentales que nous pouvons qualifier de métropolitaines, la redéfinition des politiques sociales se présentait avant tout comme un processus endogène impliquant des rapports internes. La représentation que les élites dirigeantes de ces sociétés donneront de ce processus pendant les années 1940 et 1950, sera d'inspiration fonctionnaliste : ce processus est vu comme une simple réponse aux exigences de l'industrialisation. Un tel discours ne tient évidemment pas compte du fait que diverses sociétés ont produit des modèles fort différents dans le domaine du bien-être social et qu'il n'y a pas de lien causal entre l'industrialisation et le progrès des politiques sociales; I'histoire montre que les initiatives d'avant-garde dans ce domaine sont très souvent le fruit de sociétés encore pré-industrielles. ${ }^{16}$ Pour leur part, les élites franco-québécoises, préoccupées par le maintien d'un équilibre entre facteurs de changement endogènes et exogènes, ont développé une appréciation plus subtile des enjeux de la redéfinition des politiques sociales. Celle-ci anticipe d'ailleurs les analyses récentes portant sur des sociétés périphériques où la modernisation 
joue manifestement un double rôle : elle renforce la dépendance visà-vis des métropoles extérieures en même temps qu'elle entraîne les transformations institutionnelles. ${ }^{17}$

\section{Les spécificités du contexte des années 1930-1960}

L'étude des grands débats qui ont mobilisé les Québécois au vingtième siècle fait clairement ressortir que le thème des pólitiques sociales tenait une place centrale dans la période 1930-1960. L'importance particulièrement grande que semble prendre ce sujet pour le Québec d'alors s'explique par la coïncidence suivante : au niveau des sociétés occidentales, il se produisait une révolution dans les institutions associées à la mise en œuvre du Welfare State et, à la même époque, la société franco-québécoise traversait une crise de modernisation que le problème des politiques sociales réussissait à symboliser mieux que toute autre question.

Il faut rappeler que pendant les années 1930-1960, la société franco-québécoise fut simultanément frappée par trois crises imprévisibles antérieurement. Sur un premier plan, les transformations structurelles de l'économie entrent dans une nouvelle phase qui tend à marginaliser le Québec en tant que région économique ${ }^{18}$. $\mathrm{D}^{\prime}$ autre part, la société franco-québécoise est touchée par des transformations technologiques qui ont des répercussions socio-culturelles de nature à menacer sa spécificité et sa capacité d'auto-définition. Par ailleurs, dans la logique du keynésianisme triomphant, les structures gouvernementales entrent dans une phase de centralisation des pouvoirs sous l'égide du gouvernement fédéral sapant ainsi l'influence et le prestige d'un État québécois qui avait atteint une certaine maturité dans la période de l'entre-deux-guerres.

II apparaît qu'à cause des caractéristiques particulières des politiques sociales (résumées plus haut), les élites franco-québécoises ont vu dans ce sujet un dénominateur commun reliant les plus graves problèmes de I'heure. Ainsi, dans la mesure où ces élites allaient se concentrer sur le problème du développement économique régional, elles allaient se rendre compte que les politiques sociales pouvaient influencer des mouvements de population et provoquer des changements dans le comportement de la main-d'œuvre. Les politiques sociales représentaient donc un levier essentiel pour l'orientation du développement économique en termes structurels ou régionaux. En même temps, dans la mesure où les élites se préoccupaient de l'avenir socio-culturel de la communauté franco-québécoise, elles allaient comprendre que les politiques sociales avaient aussi d'importantes implications à ce niveau : c'est une de leurs fonctions fondamentales que de redéfinir, sur de nouvelles bases, la commu- 
nauté. Enfin, dans la mesure où la question nationale était reliée au problème politique de partage des responsabilités entre les secteurs privé et public d'une part, et entre le gouvernement fédéral et les provinces d'autre part, il était devenu évident que les politiques sociales se trouvaient au cœur du problème car c'est justement dans le secteur "social» que s'étaient produits les plus importants transferts de responsabilités.

II ressort donc que la période la plus déterminante en ce qui concerne la définition et la mise en œuvre de l'État-providence à l'échelle occidentale (1930-1960) coïncide avec la phase de I'histoire québécoise au cours de laquelle les fondements de l'autodétermination et de la spécificité culturelle se trouvent le plus profondément menacés.

\section{D'une définition résiduelle à une définition institutionnelle des politiques sociales}

Même si les "paramètres" du discours québécois sur les politiques sociales que nous venons d'examiner demeurent constants tout au long de la période à l'étude, la teneur des interventions changera de façon significative. Nous chercherons maintenant à identifier les oppositions et les ruptures dans les courants de pensée qui se sont avérées les plus déterminantes pour la transformation des définitions.

\section{Étapes et catégories d'intervenants}

L'émergence au milieu des années 1960 d'un consensus sur une définition institutionnelle du bien-être social tendant à légitimer les droits de chacun à voir ses "besoins » comblés par des instances sociales responsables, marque l'aboutissement d'une longue mutation. Les phases chronologiques de cette mutation furent à peu près les mêmes au Québec que dans les sociétés ambiantes. La Crise économique d'abord et ensuite la Guerre favoriseront, chacune d'une façon particulière, le changement des perspectives en matière de bien-être social. En contrepartie, un ressac conservateur marquera l'après-guerre et les années 1950 dans la plupart des pays occidentaux. Finalement la profonde transformation culturelle qui caractérise les années 1960 favorisera l'arrivée à maturité de l'État-providence.

Nous avons vu que I'historiographie québécoise tend à lier la mutation de la définition des politiques sociales à la sécularisation de la société québécoise. Suivant cette interprétation, l'influence clé- 
ricale et la référence à la doctrine sociale de l'Église ont contribué à retarder le changement alors que les nouvelles élites séculières ont joué des rôles d'agents de transformation. Dans cette perspective, l'étape décisive d'élaboration de l'État-providence devrait coïncider avec le renversement du pouvoir clérical historiquement associé à la Révolution tranquille. Une telle interprétation ne manque pas de crédibilité en ce qui concerne certains secteurs institutionnels, celui de l'éducation par exemple. En ce qui concerne le secteur des politiques sociales par contre, elle semble résister à l'analyse. Nous verrons que l'agenda de la Révolution tranquille n'accordait aucune place à la redéfinition des politiques sociales. Cependant, les interventions en matière de bien-être qui sont allées le plus loin dans le sens d'une définition institutionnelle sont généralement venues de membres du clergé.

En analysant les courants de pensée québécois en matière de politiques sociales, il est intéressant d'examiner séparément le discours du clergé et celui des intervenants laïcs. D'abord, chacun de ces discours affiche d'importantes divisions internes; par ailleurs, le discours du clergé et celui des intervenants laïcs diffèrent du fait que le premier tend à privilégier le problème du concept de communauté alors que les interventions des seconds se concentrent sur le problème des implications économiques des politiques sociales.

\section{Discours cléricaux}

En fin de compte ce furent les milieux cléricaux beaucoup plus que les laïcs qui ont défendu une vision positive des politiques sociales pendant la période 1930-1960. Une certaine confusion plane sur le rôle de la doctrine sociale de l'église dans l'histoire des politiques sociales québécoises : cette doctrine a servi de point de référence à la fois aux partisans et aux opposants de l'extension du rôle social de l'État.

Au début des années 1930, l'Église québécoise a découvert les problèmes sociaux du prolétariat, notamment à la faveur de l'encyclique Quadragesimo Anno. À partir de ce moment, des clercs figureront autant parmi les défenseurs du développement du rôle social de l'État que parmi les opposants à cette tendance. Même si les progressistes au sein du clergé ne pouvaient s'appuyer sur une tradition de catholicisme de gauche au Québec, ils avaient accès à des sources d'inspiration européennes. Ainsi, lors du congrès social catholique de Vienne en 1929, des réformateurs autrichiens avaient avancé l'idée que le droit de propriété (le pouvoir qu'un individu avait de disposer pleinement de ses biens) devrait dépendre d'une utilisation socialement utile des biens en question. Sur ce point, 
Quadragesimo Anno tranchera en faveur de l'interprétation orthodoxe et Pie XI mettra sérieusement en garde les catholiques contre la tendance réformiste : "Socialisme religieux, socialisme chrétien, sont des contradictions : personne ne peut être en même temps bon catholique et vrai socialiste ${ }^{19}$. La tendance progressiste au sein de l'Église allait néanmoins croître en importance au cours des deux décennies suivantes.

Par exemple, quand en 1932 la commission Montpetit sur les assurances sociales, fondant ses recommandations sur une argumentation typiquement résiduelle, se déclara contre l'implantation des allocations familiales parce que celles-ci " constituent l'aveu qu'on ne peut payer des salaires suffisants pour assurer un niveau d'existence convenable $»^{20}$, le père Léon Lebel contestera les conclusions du rapport. Dans une série de neuf articles publiés dans Le Devoir, il démontrera, en se basant sur des données tant canadiennes qu'étrangères, que les salaires de la classe ouvrière se chiffraient généralement en-dessous du minimum vital. En justifiant la nécessité d'une politique d'allocations familiales, il déclarera que le rôle de l'État était de " pourvoir au bien commun, de veiller à une juste répartition des richesses de façon que toutes les classes de la société puissent se procurer les ressources nécessaires à leurs besoins ${ }^{21}$.

La contribution du catholicisme progressiste à l'éclosion d'une pensée positive en matière de politique sociale ne peut être appréciée que si on établit une distinction entre un débat essentiellement théorique touchant la doctrine et une discussion des solutions à apporter aux problèmes sociaux concrets. La doctrine catholique sur la question des politiques sociales est définie à l'aide de la philosophie de Saint Thomas d'Aquin. L'interprétation dominante dans les années 1930 voulait que la justice sociale ne soit qu'une désignation plus moderne de la justice légale; ceci impliquait que l'État devrait limiter ses interventions et favoriser l'action des individus et des corps intermédiaires dans ce domaine. Mais pendant les années 1940, et de façon plus prononcée pendant les années 1950, une nouvelle conception fera son chemin dans les milieux catholiques : la justice sociale impliquait une socialisation de la justice distributive sous l'égide de l'État ${ }^{22}$.

Pendant cette même période, ce furent les orthodoxes au sein de l'Église qui réussirent à contrôler le discours doctrinal officiel en ce qui concernait les limites du rôle de l'État. Pendant ce temps, les catholiques progressistes faisaient face à un dilemme : comment réconcilier d'une part, l'obéissance à une autorité hiérarchique relativement conservatrice et, d'autre part, l'ouverture à la culture scientifique moderne et le développement de solutions rationnelles aux problèmes sociaux ? Ils vont résoudre ce dilemme en évitant les 
questions théoriques et les débats sur l'orientation générale de la société et en adoptant une démarche empirique centrée sur des problèmes à la fois concrets et circonscrits affectant des milieux sociaux à la base. II faut souligner que ces acteurs partageaient avec leurs adversaires doctrinaires une attitude méfiante vis-à-vis le capitalisme et la conviction que les réformes institutionnelles devraient s'inspirer du catholicisme (même s'ils ne partageaient pas la même vision du catholicisme). Dans cet esprit, l'aile progressiste du clergé a appuyé dans les années 1940 l'institutionnalisation de l'assurance-chômage et des allocations familiales, ainsi que le projet d'assurance-maladie qui fit couler beaucoup d'encre pendant la guerre.

\section{Discours séculiers}

Si on examine maintenant le discours d'intervenants laïcs, il faut d'abord insister sur le fait que les rapports de classes au sein de la société franco-québécoise ont eu une importance capitale dans la période qui nous intéresse. L'acceptation d'une définition institutionnelle des politiques de bien-être devait attendre l'émergence, à la fin des années 1950 et au début des années 1960, d'une nouvelle classe moyenne constituée essentiellement de salariés en remplacement de l'ancienne composée d'entrepreneurs indépendants. Il est bien établi que les politiques sociales universalisées se sont généralisées en Occident surtout grâce à l'appui de cette nouvelle classe moyenne qui devait en tirer d'importants profits.

En attendant que le poids de ce nouvel équilibre social se fasse sentir, le discours des laïcs est plus généralement hostile face au développement du rôle social de l'État que celui du clergé. L'analyse du discours démontre que cette attitude est attribuable au fait que la question des politiques sociales est généralement associée au problème du développement économique régional et à celui de l'émancipation économique franco-québécoise. Ainsi, la référence à la pensée économique normative - un facteur limitatif des politiques sociales dans l'ensemble des sociétés - a pris un caractère " doublement " limitatif au Québec. Comme ailleurs dans les sociétés occidentales, cette référence a amené les intervenants à défendre les seuls objectifs sociaux qui n'entraient pas en conflit avec des objectifs de croissance économique. Mais au Québec, en plus d'avoir à se plier aux exigences de la croissance économique, les politiques sociales devaient tenir compte de l'idée que tous les types de croissance n'étaient pas également acceptables. L'acquisition d'une économie industrialisée autonome et diversifiée suivant des conditions spécifiquement québécoises exigeait, aux yeux d'hommes comme Esdras 
Minville et François-Albert Angers (qui comptent parmi ceux qui ont le plus écrit sur le sujet des politiques sociales), que l'État favorise le développement économique des régions du Québec avant le développement de Montréal, et les secteurs à ressources endogènes avant ceux à ressources importées (le textile par exemple). Même si ces hommes citaient à l'occasion et de façon sélective les encycliques, $c^{\prime}$ est avant tout au nom d'une logique économique qu'ils ont pris des positions restrictives en matière de politique sociale.

Quand, pendant la deuxième moitié des années 1950, les changements dans les structures socio-professionnelles et dans les rapports entre l'Entreprise et l'État, à l'échelle occidentale, auront permis l'émergence de nouvelles stratégies touchant le développement économique régional et touchant l'émancipation économique franco-québécoise, les élites accepteront plus facilement l'intégration de l'économie québécoise dans les grands réseaux d'influence nord-américains. Mais l'idéologie néo-nationaliste qui dominera la période de la Révolution tranquille (1960-1966) tiendra, tout autant que le nationalisme de l'époque précédente, à subordonner les objectifs sociaux aux objectifs économiques. Pour le gouvernement Lesage, l'égalité des chances, traduite entre autres par la démocratisation de l'accès à l'éducation, sera la politique-clé visant à résoudre les problèmes sociaux. Par ailleurs, les grandes priorités de la Révolution tranquille auront trait à l'économie ou à la promotion des nouvelles classes moyennes.

Même lorsque, peu avant les élections de 1966, un remaniement du cabinet mit enfin le rôle social de l'État au premier plan, ce rôle sera présenté avant tout dans une optique culturelle. René Lévesque renouant avec le discours duplessiste fera valoir à la conférence fédérale-provinciale de janvier 1966 que, suivant l'optique de la Commission Tremblay (1956), l'État québécois devait contrôler l'ensemble des politiques sociales dans le but de préserver la culture. Ici encore les objectifs économiques de l'État québécois paraissent sousjacents. Commentant les effets de l'établissement du régime des rentes, Nicolas Zay écrira que ce régime pourrait devenir le noyau d'un système provincial intégré de sécurité sociale mais que malheureusement, " il reflète actuellement I'ambivalence des objectifs économiques et sociaux qui ont présidé à sa naissance ${ }^{23}$.

Le peu d'importance accordé par la Révolution tranquille aux priorités sociales ne devrait pas surprendre, compte tenu du peu d'intérêt pour ce sujet parmi ceux qui avaient préparé le terrain. Des hommes comme Olivar Asselin, Jean-Charles Harvey et Jean-Marie Nadeau, généralement présentés comme les initiateurs des tendances modernistes, se sont montrés plutôt hostiles au développement du rôle social de l'État. Par ailleurs, il est remarquable de constater que si 
certains universitaires se sont intéressés au thème de la sécurité sociale pendant la guerre et encore brièvement vers 1951, on note un grand silence sur ce sujet pendant les années 1950. C'est seulement lors de la réorganisation de la revue Service social sous la direction de Guy Rocher au début de 1960, et la parution d'un numéro spécial de cette revue en juillet-août 1960 sur la sécurité sociale, qu'il est possible de constater l'apparition d'un mouvement intellectuel concerté en faveur d'une définition institutionnelle de la sécurité sociale $^{24}$. II faudra d'ailleurs attendre un certain temps avant que ce nouveau courant de pensée inspiré du Council on Social Work Education américain ne dépasse le milieu restreint de son origine.

\section{Recherche d'une forme d'intervention sociale adaptée à la spécificité québécoise}

Il faudra attendre les années 1960 avant de voir apparaître au Québec une définition institutionnelle des politiques sociales. La notion de "sécurité sociale " telle que développée dans le rapport Marsh (1943) ne suscita que très peu d'enthousiasme. Ici encore des considérations "nationales" influençaient I'analyse de la question. Au-delà du danger évident d'une mainmise fédérale sur les politiques sociales, ce qui inquiétait les progressistes autant que les conservateurs, c'était le danger que le Welfare State ne détruise les types de solidarités sociales qui caractérisaient la culture franco-québécoise (famille, paroisse) et que le prolétariat ne sente plus aucun lien organique avec la communauté nationale. On verra alors l'aile progressiste du clergé et des laïcs spécialisés dans divers domaines travailler ensemble sur un projet alternatif qui visait à introduire la modernité à la base même des milieux et des problèmes. L'intervention devenait ainsi un outil d'expression et d'action au service de la collectivité franco-québécoise plutôt qu'un facteur de dépendance. Dans un premier temps, ces pratiques concernaient l'action de spécialistes des services physiques, biologiques et humains (ingénieurs, médecins-hygiénistes, urbanistes, travailleurs sociaux) afin $\mathrm{d}^{\prime}$ identifier les problèmes sociaux et de définir des solutions. Elles impliquaient, dans un deuxième temps, la mobilisation des ressources des milieux concernés pour que ceux-ci participent dans la mesure de leurs possibilités à leur propre épanouissement. Tout en reflétant son époque, ce projet ressemble beaucoup à l'approche popularisée plus récemment sous les termes de développement communautaire. Le caractère progressiste de l'action de certaines élites des années 1940 a néanmoins été perdu de vue, cette orientation ne se conformant pas à la vision fonctionnaliste qui allait triompher au cours de la décennie suivante. 
Au début des années 1950, alors que les élites franco-québécoises commenceront à expliquer le phénomène du développement du rôle social de l'État à partir d'une interprétation fonctionnaliste d'inspiration néo-libérale, elles présenteront ce développement comme un processus inéluctablement dicté par les impératifs de l'industrialisation $^{25}$. Il est intéressant de constater que la montée de ce schéma conceptuel coïncidera au Québec avec un déclin des politiques sociales en tant que préoccupation idéologique et en tant que priorité pour les partis politiques. II faudra attendre la seconde moitié des années 1960 pour que ce sujet regagne la place qu'il avait occupée parmi les grandes préoccupations des années 1930 et 1940.

\section{L'équilibre des facteurs exogènes et endogènes dans I'histoire des politiques sociales}

En conclusion nous posons la question suivante : à la lumière des éléments développés plus haut, quelle contribution l'approche historique apporte-t-elle au problème de la définition du sens que doivent prendre les politiques sociales dans les sociétés en rapide évolution?

II nous semble que la révélation la plus significative sur cette question que nous apporte l'analyse de l'expérience québécoise est de voir comment les conditions spécifiques à une société particulière peuvent modifier la problématique. Dans le feu de l'action, les contemporains considèrent généralement que les grands courants de pensée, les grandes tendances structurelles et culturelles qui se déploient à l'échelle occidentale, déterminent à eux seuls les voies du changement. L'historien qui réexamine les événements après le passage du temps, vise pour sa part à mettre en évidence les différences importantes qui marquent l'expérience de chaque société et il est poussé à en rechercher les causes.

La principale faiblesse que nous avons constatée dans l'historiographie québécoise des politiques sociales est une tendance à insister sur l'inévitabilité d'une convergence avec un modèle occidental unique en ce domaine. Nous avons vu toute l'importance des facteurs exogènes dans le processus de redéfinition des politiques sociales. Les principaux paramètres du discours sont communs aux sociétés occidentales et les grands courants de pensée comme la doctrine sociale de l'Église, le courant keynésien et le courant développemental du Council on Social Work Education viennent de l'extérieur. Par contre, il ne faut pas négliger le sens des interventions des diverses élites d'ici qui ont toutes en commun la recherche d'une conciliation des enjeux des politiques sociales avec une probléma- 
tique de développement spécifiquement québécoise, soit au niveau de la définition du sens de "communauté », soit au niveau du développement économique.

\section{Notes}

1 Peter Southam, "Modernisation", "Question nationale " et influences exogènes : Le discours élitaire sur les politiques sociales au Québec, 19301960, Thèse de doctorat, Université Laval, 1987, 447 p.

${ }^{2}$ Nous référons ici aux concepts résiduel et institutionnel tels que développés par L. Wilensky et Charles N. Lebeaux : "Deux conceptions dominantes s'imposent actuellement aux États-Unis concernant la notion de politique sociale; l'une se veut résiduelle et l'autre, institutionnelle. La première conçoit le rôle des politiques sociales comme devant être palliatives en regard des structures sociales habituelles telles que la famille, les échanges marchands, les services, etc. La deuxième conception s'oppose à la première en ce qu'elle suggère des services sociaux universalisés s'inscrivant à l'intérieur des circuits normaux et habituels des sociétés industrielles modernes ". (Industrial Society and Social Welfare, p. 138).

${ }^{3}$ Cette interprétation trouve sa définition la plus élaborée dans Guy Rocher, Le Québec en mutation, Montréal, 1973, 345 p.

${ }^{4}$ Rapport du Comité d'étude sur l'assistance publique, Québec, 1963, 230 p.

${ }^{5}$ Id., p. 108.

${ }^{6}$ Yves Vaillancourt, L'évolution des politiques sociales au Québec : 1940-1960, Montréal, 1988, $513 \mathrm{p}$.

${ }^{7}$ Id., p. 484.

${ }^{8}$ Id., p. 485.

${ }^{9}$ Gary Caldwell et B. Dan Czarnocki, « Un rattrapage raté, le changement social dans le Québec d'après-guerre 1950-1974», Recherches sociographiques, vol. 18, n 1, janvier-avril $1977:$ 9-58.

${ }^{10}$ Fernand Ouellet, "La question sociale au Québec, 1880-1930. Perspectives historiographiques et critiques " in Ginette Kurgan-van Hentenryk, éd., La question sociale en Belgique et au Canada : XIX ${ }^{\mathrm{e}}-X X^{\mathrm{e}}$ siècles, Bruxelles, 1988, p. 56: Paul-André Linteau et al., Histoire du Québec contemporain : Le Québec depuis 1930, Montréal, 1986, p. 579.

${ }^{11}$ Un exemple parmi beaucoup d'autres, trop nombreux pour être énumérés ici, concerne les coûts d'hospitalisation. Dans les années 1930, comparativement à l'Ontario, une proportion nettement supérieure des patients d'institutions de santé du Québec bénéficiaient d'un régime de gratuité grâce à des initiatives de l'État.

12 Paul-André Linteau et al., Histoire du Québec contemporain, p. 579.

${ }^{13}$ David Wolfe, The Delicate Balance: The Changing Role of the State in Canada, Thèse de doctorat, Université de Toronto, 1980, p. 361; Anne Légaré et Nicole Morf, La société distincte de l'État: Québec-Canada 1930-1980, Ville LaSalle, 1989, 237 p.; Dennis Guest, The Emergence of Social Security in Canada, Vancouver, 1980, 259 p.

${ }^{14}$ Hugh Heclo, Modern Social Politics in Britain and Sweden : From Relief to Income Maintenance, New Haven, 1974, p. 312. Voir aussi p. 97-118. 
${ }^{15}$ Robert Pinker, The Idea of Welfare, London, $1979: 40-47$.

${ }^{16}$ Hugh Heclo, Modern Social Politics in Britain and Sweden; Daniel Levine, "Conservatism and Tradition in Danish Social Welfare Legislation, 18901933 : A comparative View ", Comparative Studies in Society and History, vol. 20, n' 1, janvier 1978 : 54-69.

${ }^{17}$ Peter Schneider et al., "Modernization and Development : The Role of Regional Elites and Noncorporate Groups in the European Mediterranean ", Comparative Studies in Society and History, vol. 14, $\mathrm{n}^{\circ}$ 3, juin 1972 : 328-350.

${ }^{18}$ Joseph $\mathrm{H}$. Chung, "La nature du déclin économique de la région de Montréal " in Rodrigue Tremblay, dir., L'économie québécoise, Montréal, 1976 : 427-438.

${ }^{19} \mathrm{Pie} \mathrm{XI}$, L'encyclique Quadragesimo Anno: Sur la restauration de l'ordre social, Montréal, s.d., p. 50.

${ }^{20}$ Québec, Commission des assurances sociales, p. 98.

${ }^{21}$ Le Devoir, 25 avril 1932.

${ }^{22}$ L'énoncé le plus clair de ce principe viendra de la plume de Gilles-M. Bélanger, o.p., "La sécurité sociale en regard de l'enseignement social de l'Église ", Service social, vol. 9, n² 2, juillet-août 1960.

${ }^{23}$ Nicolas Zay, "Sécurité sociale et bien-être ", Relations, octobre 1966, p. 279.

${ }^{24}$ Voir "Une orientation nouvelle ", Service social, vol. 9, $\mathrm{n}^{\circ} 1$, janvier 1960; "La sécurité sociale", Service social, vol. 9, n 2, juillet-août 1960.

${ }^{25}$ Voir Maurice Lamontagne, Le fédéralisme canadien : évolution et problèmes, Québec, 1954, 298 p. 